\title{
Limitada producción científica de la comunidad universitaria: Rol de los incentivos
}

\author{
Limited scientific production of the university community: Role of incentives
}

\section{Sr. Editor:}

La reducida producción nacional científica de las universidades comparada con otras regiones de Latinoamérica es alarmante. Solo el $1 \%$ es considerada como visibilidad científica óptima (1). En la actualidad, los artículos científicos son considerados como los productos de proyectos de investigaciones, sin embargo, para lograr la creación del estado del arte el punto de partida debe surgir de la universidad con un intenso programa de asesorías y acompañamiento no solo académico sino también, económico $(2,3)$.

El manejo de los recursos económicos en investigación en las entidades universitarias de Latinoamérica no está siendo reflejado en ningún estudio ni análisis y en el país las aproximaciones sobre aportes económicos a los estudiantes e investigadores aún no tienen impacto en la producción científica (4). Según el censo nacional de investigación realizado por el Consejo Nacional de Ciencia, Tecnología e Innovación Tecnológica (CONCYTEC) en el año 2015, la reducción de publicación de artículos científicos se relacionó con la remuneración de los docentes investigadores y del personal de apoyo (5). Dicha información resalta la importancia de los incentivos económicos enfocada en el desarrollo de cualidades y habilidades investigativas con la finalidad de generar visibilidad científica a nivel nacional e internacional.

La problemática que engloba la escasez de producción científica, en los últimos años ha establecido medidas sobre apoyos económicos mediante centros y redes de investigación que financien la publicación de artículos científicos (6). La publicación de artículos científicos representa la parte final del estado del arte en la investigación, no obstante, en el año 2016 en el Perú las universidades solo publicaron tres artículos científicos en revistas indizadas revelando un margen inferior al del continente africano con 10 artículos publicados anualmente (7). Si relacionamos las publicaciones científicas con la inversión, países como Brasil, México y Argentina son los únicos que han demostrado gastos en investigación proporcionados con la producción científica en donde se reconoce a la participación de docentes investigadores remunerados para alcanzar publicaciones científicas en revistas de alto impacto (8).

En conclusión, la inversión económica correctamente distribuida en las investigaciones de las universidades peruanas puede marcar un avance en el incremento de la producción científica a nivel de docentes y estudiantes. Además, la generación del impacto científico debe ser valorado por las universidades nacionales y así brindar la importancia necesaria de la visibilidad académica de los productos investigativos hacia otras comunidades universitarias alrededor del mundo.

\section{Andrea Mirella Arpita Rojas 1,a;2}

\section{Correspondencia:}

Andrea Mirella Arpita Rojas

Jr. Buenaventura Aguirre 975

San Juan de Miraflores, Lima, Perú.

Celular 51955566575

Correo electrónico: andrea.arpita@unmsm.edu.pe

\footnotetext{
Maestría en Salud Pública. Universidad Nacional Mayor de San Marcos. Lima, Perú.

Coordinación de investigación. Universidad Seminario Evangélico de Lima. Lima, Perú.

Egresada.
} 


\section{REFERENCIAS BIBLIOGRÁFICAS}

1. Bornmann L, Mutz R. Growth rates of modern science: a bibliometric analysis based on the number of publications and cited references. J Assoc Inform Science Technol. 2015; 66(11):2215-2222.

2. Moquillaza V. Producción científica asociada al gasto e inversión en investigación en universidades peruanas. An Fac med. 2019; 80(1):56-59.

3. Alarcón C, Quezada M. Publicación de artículos científicos por asesores de tesis de una Facultad de Medicina. Rev Med Hered. 2018; 29(1):152-157.

4. Nieto W, Fernández J, Taype A, Pacheco J, Mayta P. Incentivos por publicación científica en universidades peruanas que cuentan con escuelas de medicina, 2017. Rev peru med exp salud publica. 2018; 35(2):354-356.

5. Consejo Nacional de Ciencia, Tecnología e Innovación Tecnológica. I Censo Nacional de Investigación y Desarrollo a Centros de Investigación. Lima: CONCYTEC; 2016. (Citado el 30 de mayo del 2019) Disponible en: http://portal.concytec.gob. pe/index.php/publicaciones/censo-nacional-id
6. Farrokhyar F, Bianco D, Dao D, et al. Impact of research investment on scientific productivity of junior researchers. Transl Behav Med. 2016; 6(4):659-668.

7. Obuku E, Lavis J, Kinengyere A, et al. Academic research productivity of post-graduate students at Makerere University College of Health Sciences, Uganda, from 1996 to 2010: a retrospective review. Health Res Policy Syst. 2017; 15(1):30.

8. Robles R, Vela F, Huapaya O, Chacón H. Relación entre el gasto en investigación y desarrollo con la producción científica en el Perú. An Fac Med. 2015; 76(4):469-470.

Recibido: 21/06/2019 